\title{
Joint Power Allocation and Scheduling of Multi-antenna OFDM System in Broadcast Channel
}

\author{
Feng She, Wen Chen, Hanwen Luo, Tingshan Huang, and Xinbing Wang \\ Department of Electronic Engineering, Shanghai Jiaotong University, China 200030 \\ Email: \{shefeng630;wenchen\}@ sjtu.edu.cn
}

\begin{abstract}
This paper considers the general multiuser downlink scheduling problem and power minimization with multiuser rate constraints. We present joint user selection algorithms for DPC, ZF-DPC, ZFBF and TDMA for multi-antenna OFDM system in broadcast channels, and we also present a practical waterfilling solution in this paper. By the selected users with the consideration of fairness, we derive the power optimization algorithm with multiuser rate constraints. Simulation results show that the presented user scheduling algorithms and power minimization algorithms can achieve good power performance. Meanwhile, simulation results also show that the scheduling algorithm can guarantee fairness.
\end{abstract}

\section{INTRODUCTION}

MIMO-OFDM is now a popular topic in both literature and industry and has very high potential for the future wide band wireless communication systems. In practical systems, it is well known that multiple antennas can be easily deployed at base station in cellular systems. However, mobile terminals usually have a small number of antennas due to the size and cost constraint. Thus, it may appear that we do not obtain significant capacity benefit from the multiple transmit antennas. This is true with the transmit strategy of time division multiple access (TDMA) [1]. To solve the problem, multiuser must be served simultaneously. One way to accomplish this is called dirty paper coding (DPC), which is a multiuser encoding strategy based on interference presubtraction [2]. Since DPC is of high complexity, [3] presents zero forcing dirty paper coding (ZF-DPC) as a suboptimal solution, while the complexity is still unacceptable. As a much simple transmit strategy, zero forcing beamforming (ZFBF) has been proposed for space division multiple access (SDMA) to remove the cochannel interference in MIMO downlink systems [5], [6].

Various researchers have investigated that the sum capacity gain is achievable in the above described systems by simultaneously transmitting symbols to several users. In particular, Jindal and Goldsmith [4] show that the sum capacity gain over TDMA strategy is approximately $\min \left\{N_{t}, K\right\}$, i.e., the minimum of the number of transmit antennas and the number of users. In information theoretic terms, multiuser downlink channel may be modeled as a multiuser antenna broadcast channel (BC) [3], [7], [9]-[11], which is a typical environment encountered in multiuser communications, such as wireless LAN and cellular systems. In broadcast channel (BC) and multi-access channel (MAC), power control is an important mechanism to solve the near-far problem. In multi-antenna OFDM systems, the optimal power allocation for the desired rate in the achievable rate region is not clearly known until now. Most literatures on this topic only address the maximum sum rate problem when each user has his own power budget. In other words, most previous works are concentrated on the sum capacity, or maximum total throughput, of BC or MAC. Though this is an extremely useful metric, the resulting rate allocation to receivers appear to be those users with strong channels are typically allocated more rate than those with weaker channels, and some users may not be allocated any rate at all. This may be undesirable in certain systems [8]. In most practical cases, each user has a desired data rate and likes to achieve it within minimum power. Thus, it is an important problem to guarantee all the user's desired data rates while consuming minimum power. The motivation of the problem is power saving and reducing the co-channel interference to neighbor cells.

The focus of this paper is the sum power minimization problem for multi-antenna OFDM systems in BC downlink with user selection. In this paper, we study the optimal transmit schemes for DPC, ZF-DPC, ZF techniques with optimal power allocation algorithm by multi-user rate constraints. We investigate the power duality of BC and MAC, and we also propose the minimum power allocation solution for different transmit strategies. We derive the close form of minimum power allocation for DPC, ZF-DPC, ZFBF in MIMO-OFDM systems, and give the practical solution of waterfilling for the minimization power allocation algorithms. We analyze the power duality between MAC and BC for DPC, ZF-DPC, ZFBF, and develop the user scheduling algorithm for DPC, ZF-DPC, ZFBF with the consideration of fairness.

Notation used in this paper are as follows: $(\cdot)^{T}$ denotes matrix transposition, $(\cdot)^{\mathbf{H}}$ denotes matrix conjugate-transposition, $E[\cdot]$ denotes statistical expectation, and $\|\cdot\|^{2}$ denotes the mean square norm of a vector.

\section{Multi-Antenna OFDM BROAdCAST CHANnEL}

Fig. 1 shows the multi-user MIMO-OFDM system in broadcast channel. We assume that the transmitter can get perfect channel knowledge. Thus, the transmitter schedules a set of users to be served and employ power allocation on each subcarrier. We focus on quasi-static channels, where the channel is fixed over the time period of interest (i.e., over the period of the delay constraint). In multiuser MIMO-OFDM system, we consider a wireless communication system with one base station and $K$ users. The base station is with $N_{t}$ 


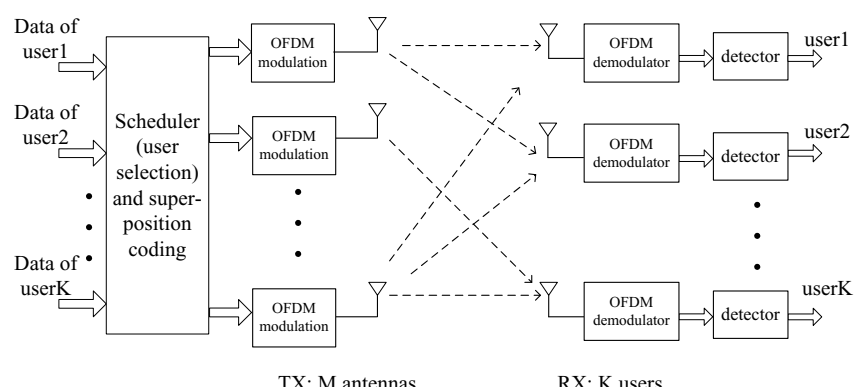

Fig. 1. Block diagram of multi-antenna OFDM system in broadcast channel

transmit antennas, and each of the users has single receive antenna. In an OFDM symbol, the number of total subcarrier is $N_{c}$. Thus the channel between the base station and user $k$ on subcarrier $n$ can be characterized by $1 \times N_{t}$ matrix $h_{i, n}$. For every user $i$, the base station transmits data $x_{i, n}$ after a $N_{t} \times 1$ dimension beamformer $v_{k, n}$ on subcarrier $n$. The signal at the $i$ th user's receiver on the $n$th subcarrier is

$$
\boldsymbol{y}_{i, n}=h_{i, n} \sum_{j=1}^{K} \sqrt{p_{j, n}} v_{j, n} x_{j, n}+w_{i, n} .
$$

where $w_{i, n}$ is white Gaussian noise at the $i$ th user on subcarrier $n$ and $p_{j, n}$ is the power allocated to the $i$ th user on the $n$th subcarrier.

\section{A. Multi-antenna DPC, ZF-DPC and ZFBF}

1) Dirty paper coding: It is now well known that dirty paper coding (DPC) achieves the sum capacity of the multiple antenna broadcast channel as well as the full capacity region [13]. DPC is a precoding technique that precancel interference at the transmitter [2], [3], [11], [13], [16].

2) Zero forcing beamforming $(Z F B F)$ in MISO BC: As mentioned above, multiple transmit antennas can potentially yield an $N_{t}$-fold increase in the sum capacity. [1] showed that employing ZFBF to a set of $N_{t}$ nearly orthogonal users with large channel norms is asymptotically optimal as the number of users grows large.

3) Zero forcing dirty paper coding (ZF-DPC) in MISO BC: Finding simple ways to achieve performance close to DPC capacity is still of great interest for the MIMO enhanced broadcast channel [7], [12]. In [3], a simple approach based on $\mathrm{QR}$ decomposition is proposed to optimize the sum rate for channel matrices with full row rank. It is referred as zero forcing dirty paper coding (ZF-DPC). Zero forcing method provides efficient, closed form solutions that yield a reasonable tradeoff between performance and computational complexity [6] and can easily be adopted in MIMO-OFDM systems.

\section{OPTIMAL PROBLEM AND SOLUTION}

The work of DPC power allocation has been well done in [15]. Therefore, we focus on the power allocation optimization on ZF-DPC, ZFBF, and the results can be extended to MAC naturely by power duality.

\section{A. Optimal problem}

We consider the case that each receiver has only one antenna. The optimal power allocation problem is to determine the minimum required transmit power to achieve a given rate vector $R$, which is an optimization problem as follows

$$
\begin{cases}\min & \sum_{j=1}^{K} \sum_{n=1}^{N_{c}} p_{j, n} \\ \text { s.t. } & \sum_{n=1}^{N_{c}} \log _{2}\left(1+d_{1, n} p_{1, n}\right) \geq R_{1}, \\ & \vdots \\ & \sum_{n=1}^{N_{c}} \log _{2}\left(1+d_{K, n} p_{K, n}\right) \geq R_{K},\end{cases}
$$

where $p_{j, n}$ is the power allocated on the $n$th subcarrier of user $j$, and $d_{j, n}$ denotes the channel gain of user $j$ on the $n$th subcarrier.

Lemma 1: For a Gaussian $\mathrm{BC}$ with channel gain $d=$ $\left[d_{1}, d_{2}, \cdots, d_{K}\right]$, and noise variance 1 , the optimal power of $p_{j, n}$ can be noted as

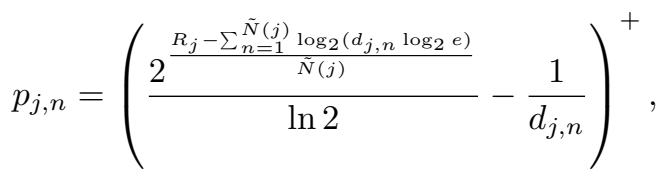

where $(x)^{+}$denotes $\max \{x, 0\}$, and $\tilde{N}(j)$ is the number of subcarriers allocated to user $j$.

The proof is omitted due to limited paper length.

For practical solution of power allocation, some subcarrier will not be allocated any power. Thus, in (2), $N_{c}$ will be substituted as $\tilde{N}(j) \leq N_{c}$ for the $j$ th user. The work of determination of $\tilde{N}(j)$ will be done in the next subsection.

\section{B. Practical solution of waterfilling}

Our goal is to minimize the transmit power subject to each user's rate constraint. We employ water-filling based algorithm to get the optimal solution. By (3), we carry out the power allocation for each user. The key work of waterfilling is to determine the $\tilde{N}(j)$ in (3). After getting $\tilde{N}(j)$, the $p_{j, n}$ can be easily computed by (3).

Let $p_{j, n}=\left(\mu(j)-b_{j, n}\right)^{+}$denote the power of the $j$ th user on the $n$th subcarrier, where $\mu(j)$ is the water level of user $j$. Then, the waterfilling solution can be numerically evaluated in practice as follows.

S1) Set $\tilde{N}(j)=N(j)$, where $N(j)$ is the number of allocated number of user $j$, and sort the set of $d_{i, n}$, for $n \in[1, N(j)]$, such that $d_{i, n}$ is in decreasing order. Set $\tilde{\mu}(\tilde{N}(j))=2^{\frac{R_{j}-\sum_{n=1}^{\tilde{N}(j)} \log _{2}\left(d_{j, n} \log _{2} e\right)}{\tilde{N}(j)}} / \ln 2$.

S2) If $\tilde{\mu}(\tilde{N}(j))-1 / d_{j, \tilde{N}(j)}<0$, then $\tilde{N}(j)=N(j)$, and go to S3. Otherwise, set $\tilde{N}(j)=\tilde{N}(j)-1$, and repeat step S2.

S3) Find the water level $\mu(n)(j)$ of user $j$ as: 


$$
\mu(j)=2^{\frac{R_{j}-\sum_{n=1}^{\tilde{N}(j)} \log _{2}\left(d_{j, n} \log _{2} e\right)}{\tilde{N}(j)}} / \ln 2 .
$$

\section{MULTi-USER SCHEDULE FOR MIMO OFDM BROADCAST CHANNEL}

In this section, we develop user group selection algorithms for ZF-DPC, ZFBF in MIMO-OFDM systems.

\section{A. Algorithm 1 (for DPC, ZF-DPC)}

Let $T$ be the user set, and $N$ be subcarrier set, $h_{k}\left(N_{s}\right)$ be the channel vector of the $k$ th user on the $N_{s}$ th subcarrier. $S\left(N_{s}\right)$ is the selected user set on the $N_{s}$ th subcarrier and $N_{c}$ is the total number of subcarriers, $N_{K}$ is the number of total candidate users.

S1) $\bullet$ Set $T=\left\{1, \cdots, N_{K}\right\}, N=\left\{1, \cdots, N_{c}\right\}$, and $n=1$. - Let $S\left(N_{s}\right)=\phi$ (empty set), $N_{s} \in\left\{1, \cdots, N_{c}\right\}$.

S2) If $T=\phi$ (empty set), then set $T=\left\{1, \cdots, N_{K}\right\}$. Let $r_{1, k}\left(N_{s}\right)=h_{k}\left(N_{s}\right) h_{k}\left(N_{s}\right)^{H}$, where $k \in T$ and $N_{s} \in N$. Find a user $s_{1}\left(N_{s}\right)$ such that

$$
s_{1}=\arg \max _{\left\{k \in T, N_{s} \in N\right\}} r_{1, k}\left(N_{s}\right) .
$$

Set $S\left(N_{s}\right)=S\left(N_{s}\right) \bigcup\left\{s_{1}\right\}, T=T-\left\{s_{1}\right\}$.

S3) While $n \leq N_{t}$, increase $n$ by 1. Project each remaining channel vector onto the orthogonal complement of the sub-space spanned by the channels of the selected users. By [17], the projector matrix is

$$
\begin{aligned}
& P_{n}\left(N_{s}\right)^{\perp}=I_{N_{t}}-H\left(S_{n-1}\left(N_{s}\right)\right)^{H} \\
& \left(H\left(S_{n-1}\left(N_{s}\right)\right) H\left(S_{n-1}\left(N_{s}\right)\right)^{H}\right)^{-1} H\left(S_{n-1}\left(N_{s}\right)\right),
\end{aligned}
$$

where $I_{N_{t}}$ is the $N_{t} \times N_{t}$ identity matrix, and $H\left(S_{n-1}\right)$ denotes the channel vectors of the users selected in the first $n-1$ steps on the $N_{s}$ th subcarrier.

$$
H\left(S_{n-1}\right)=\left[\begin{array}{llll}
h_{s_{1}}^{H} & h_{s_{2}}^{H} & \cdots & h_{s_{n-1}}^{H}
\end{array}\right]
$$

Let $r_{n, k}\left(N_{s}\right)=\left|h_{k}\left(N_{s}\right) P_{n}\left(N_{s}\right)^{\perp}\right|^{2}$. Due to the idempotence of $P_{n}\left(N_{s}\right)^{\perp}$, we have

$$
r_{n, k}\left(N_{s}\right)=h_{k}\left(N_{s}\right) P_{n}\left(N_{s}\right)^{\perp} h_{k}\left(N_{s}\right)^{H} .
$$

Find a user $s_{n}$ on the $N_{s}$ th subcarrier such that

$$
s_{n}=\arg \max r_{n, k}\left(N_{s}\right) .
$$

Set $S\left(N_{s}\right)=S\left(N_{s}\right) \bigcup\left\{s_{n}\right\}$, and $T=T-\left\{s_{n}\right\}$.

S4) Let $N=N-\left\{N_{s}\right\}, n=1$. While $N \neq \phi$ (empty set), go to step2.

S5) Beamforming on each subcarrier: On each subcarrier, let $v=Q^{H}$, where $H\left(S\left(N_{s}\right)\right)=L Q$ is QR-type decomposition of $H\left(S\left(N_{s}\right)\right)$.

S6) Applying DP coding on the rows of $L$.

S7) Power allocation: waterfilling across subcarriers and data streams.

\section{B. Algorithm2 (for ZFBF)}

S1) Same as Algorithm 1.

S2) Same as Algorithm 2 excerpt that

$$
s_{n}=\arg \max _{s_{n} \in T} R_{Z F}\left(S\left(N_{s}\right) \bigcup\left\{s_{n}\right\}\right) .
$$

S3) While $n \leq N_{t}$ : Increase $n$ by 1 . Denote the achievable rate by $R_{Z F}\left(S\left(N_{s}\right)\right)$. Find a user $s_{n}$, such that

$$
s_{n}=\arg \max _{s_{n} \in T} R_{Z F}\left(S\left(N_{s}\right) \bigcup\left\{s_{n}\right\}\right) .
$$

Set $S\left(N_{s}\right)=S\left(N_{s}\right) \bigcup\left\{s_{n}\right\}$, and $T=T-\left\{s_{n}\right\}$.

S4) If $R_{Z F}\left(S_{n}\right)_{\max }<R_{Z F}\left(S_{n-1}\right)_{\max }$, set $S\left(N_{S}\right)=$ $S\left(N_{S}\right)-\left\{s_{n}\right\}$.

S5) Set $N=N-\left\{N_{s}\right\}$.

S6) If $N \neq \phi$ (empty set), set $n=1$, and go to step 2 .

S7) Perform beamforming on each subcarrier: On each subcarrier, let

$$
v=H\left(S\left(N_{s}\right)\right)^{H}\left(H\left(S\left(N_{s}\right)\right) H\left(S\left(N_{s}\right)\right)^{H}\right)^{-1},
$$

where $H\left(S\left(N_{s}\right)\right)=L Q$ is $\mathrm{QR}$ decomposition of $H\left(S\left(N_{s}\right)\right)$.

S8) Power allocation: Same as the S7) in Algorithm 1.

\section{Algorithm3 (for TDMA)}

In TDMA, there is only one data stream to be sent on each subcarrier. Thus, we only need to select the strongest channel for each subcarrier. Firstly, we select a user $s_{1}$ with strongest channel gain on one subcarrier, and then set the initial user set $T=\left\{1, \cdots, N_{K}\right\}-\left\{s_{1}\right\}$. Then repeat the above selection processing until each subcarrier is allocated to some users. The last work is to do waterfilling across each subcarrier.

\section{Simulation Results and Discussion}

We assume that the discrete-time channel impulse response is generated according to the Hiperlan2 Channel Model $\mathrm{C}$ in [18]. The channels between different transmit and receive antennas are assumed to be independent. The transmitter power is allocated across subcarriers by waterfilling principle presented in part III. The receiver used linear decoder with perfect channel knowledge. It is also assumed that the transmitter has perfect knowledge of channel state information.

A. Experiment 1: The number of transmitter antenna is 4, and each user is equipped with one receive antenna. The number of subcarriers is 64 and the cyclic prefix length is 16. The Rate $_{0}$ in Fig. 2 is randomly created which ranges from 0.01 to 0.16 bits. The rate of each user is the multiple of Rate $_{0}$, and the number of users in this experiment is 80 . Fig. 2 shows the needed power of the power allocation algorithms.

B. Experiment 2: The number of transmit antennas is 8 and the OFDM configuration is the same as Experiment 1. The way of rate creation is also the same as that of Experiment 1. By 10000 times channel realization, we get the result as Fig. 3 . Compared with Fig. 2, we can see that the increment of transmit antenna can reduce the total transmit power. 


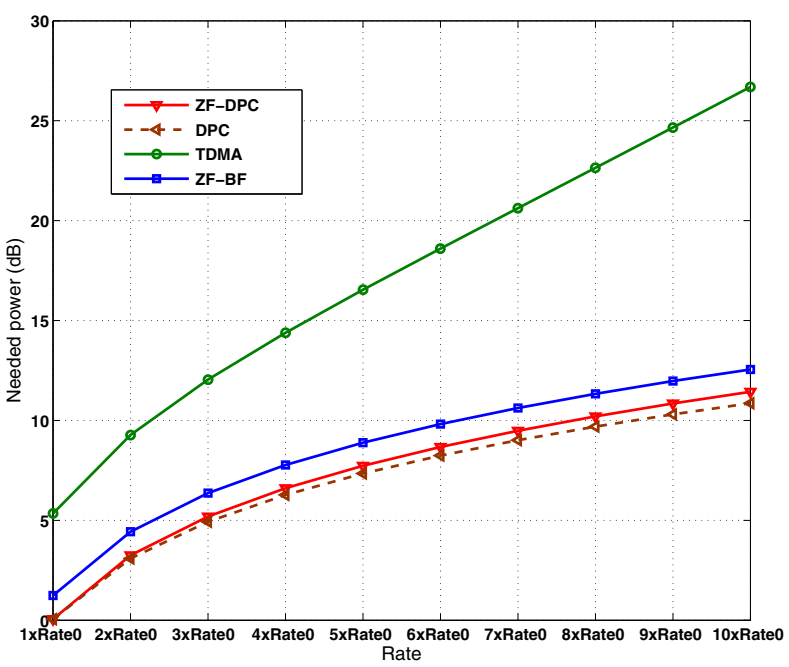

Fig. 2. Sum needed power of different rate required with 4 transmit antenna.

C. Experiment 3: This is about the influence of selection algorithm with the consideration of fairness. By 10000 times of channel realization, we can see from Fig. 4 that the needed power of TDMA, DPC, ZF-DPC and ZFBF. Comparing with Fig. 2, we can see that the needed power of algorithm without fairness consideration is less than that with consideration of fairness.

D. Experiment 4: The fourth experiment is about the fairness of user selection. In this experiment, we consider the subcarrier 1024, and the user scheduling scheme in Algorithm 2. We consider 100 users in this experiment. Without loss of generality, We only consider the scenario of ZFBF. The results of ZF-DPC would be similar to that of ZFBF. We test the scheduling algorithm for 10000 times. Fig. 5 shows that by Algorithm 2, the selected frequency of each user is very close. Thus, by the algorithm 2, the system can achieve considerable fairness, and we can also look this experiment as the duality of Experiment 2.

E. Experiment 5: The rate of each user in this experiment is randomly created which ranges from 0.0156 to 0.25 . Without loss of generality, the number of users in each simulation scenario is the multiple of 64 . We also assume that there are 64 users served at one time slot.

F. Experiment 6: The rate of each user is randomly created which ranges from 1 to 8 . Here, we assume that the number of user is 64 . We compare the total power consumption of TDMA, ZFBF and ZF-DPC. Fig. 7 shows that the total power of the three scenario is same when the number of the transmit antenna is one.

\section{CONCLUSION}

In this paper, we consider the user scheduling and power allocation for MIMO-OFDM system in broadcast channel. We propose user scheduling algorithms for ZF-DPC, DPC and ZFBF in multi-antenna OFDM system. We also present the

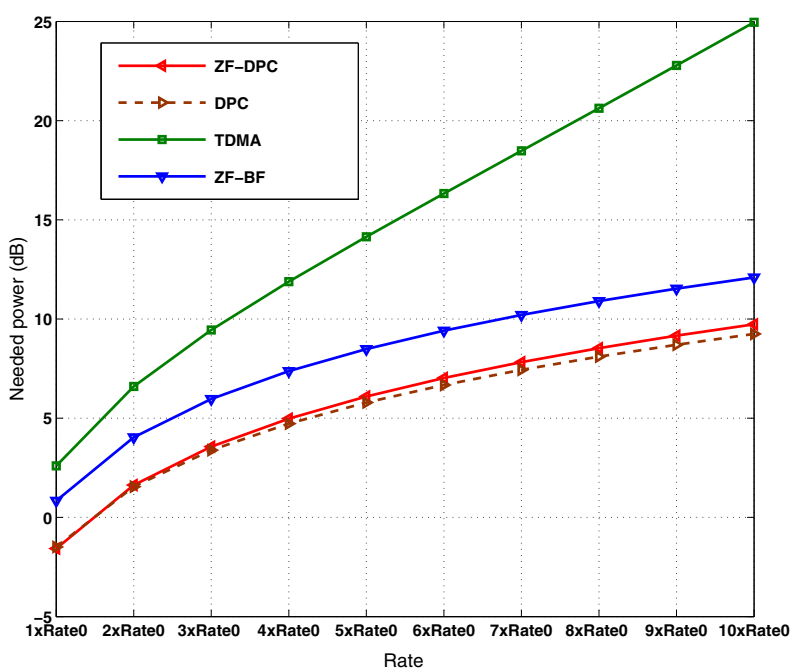

Fig. 3. Sum needed power of different rate required with 8 transmit antenna.

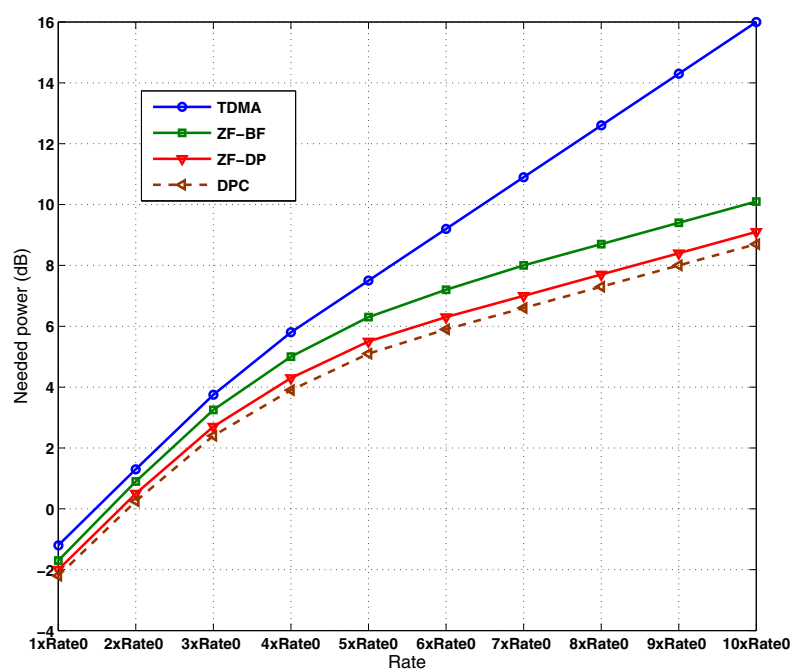

Fig. 4. Sum needed power of different rate required without consideration of fairness.

general solution of minimization of transmitter power. We have examined the transmit strategies for multi-antenna $\mathrm{BC}$ with large number of users. We have show that ZFBF can achieve considerable performance but with much lower complexity. The proposed user scheduling algorithms is indeed with good fairness performance for different transmit strategies. We also show that the needed power will be reduced with the increment of the number of users. This is because that with large number of users, the transmitter can choose users with good channel condition including channel gain and orthogonality among user channel vectors. Although we focus on $\mathrm{BC}$, we also mentioned the duality between $\mathrm{BC}$ and MAC. By this duality, all the result in this paper is directly applicable to the MAC 


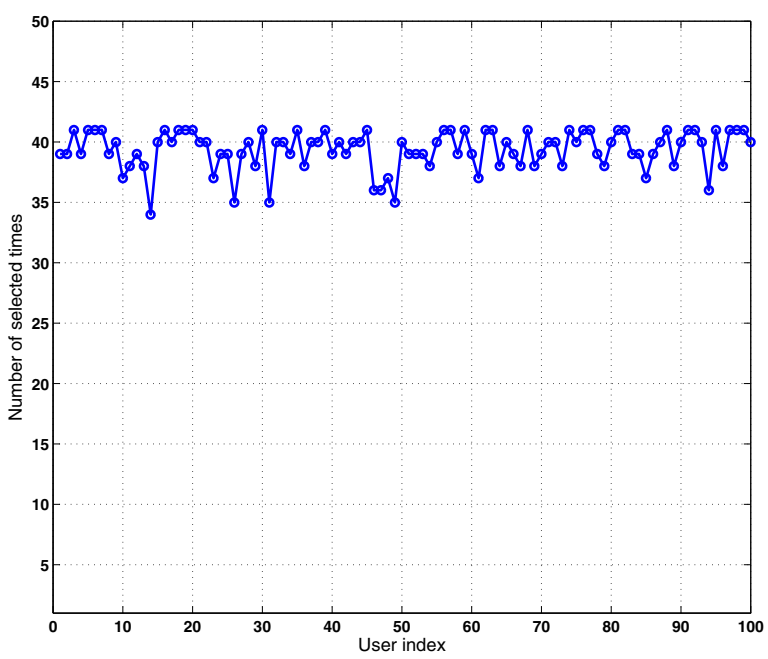

Fig. 5. The performance of fairness by algorithm 2 of ZFBF

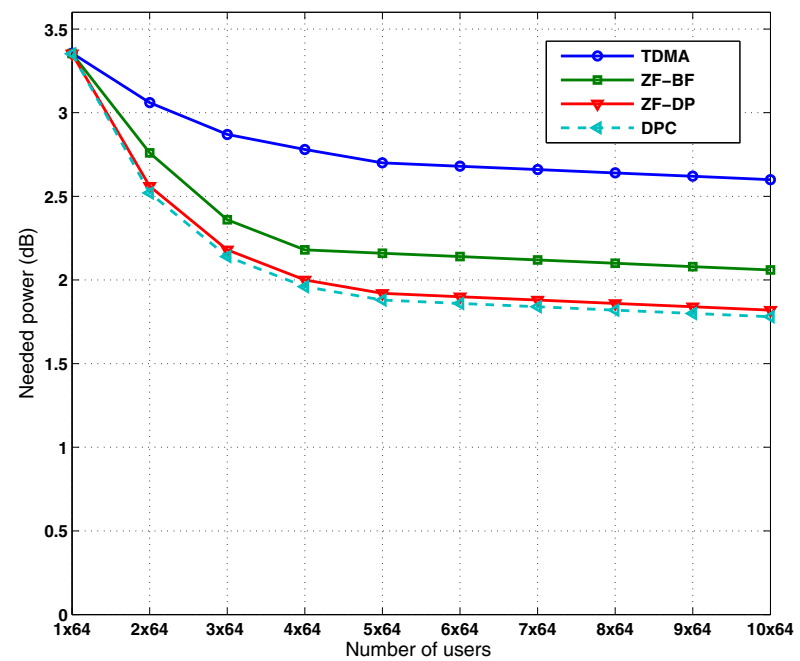

Fig. 6. The effect of multiuser diversity.

scenario.

\section{ACKNOWLEDGMENT}

This work is supported by NSF China \#60572157, \#60672067, by Cultivation Fund of the Key Scientific and Technical Innovation Project, Ministry of Education of China \#706022, by Program for New Century Excellent Talents in University \#NCET-06-0386, and by PUJIANG Talents \#07PJ4046.

\section{REFERENCES}

[1] T. Yoo, A. Goldsmith, "On the optimality of multi-antenna broadcast scheduling using zero-forcing beamforming," IEEE J. Sel. Areas Commun., vol. 24, no. 3, pp. 528-541. May. 2006.

[2] M. H. M. Costa, "Writing on dirty paper," IEEE trans. Inf.Theory, vol. 29, no. 3, pp. 439-441. Mar. 1983

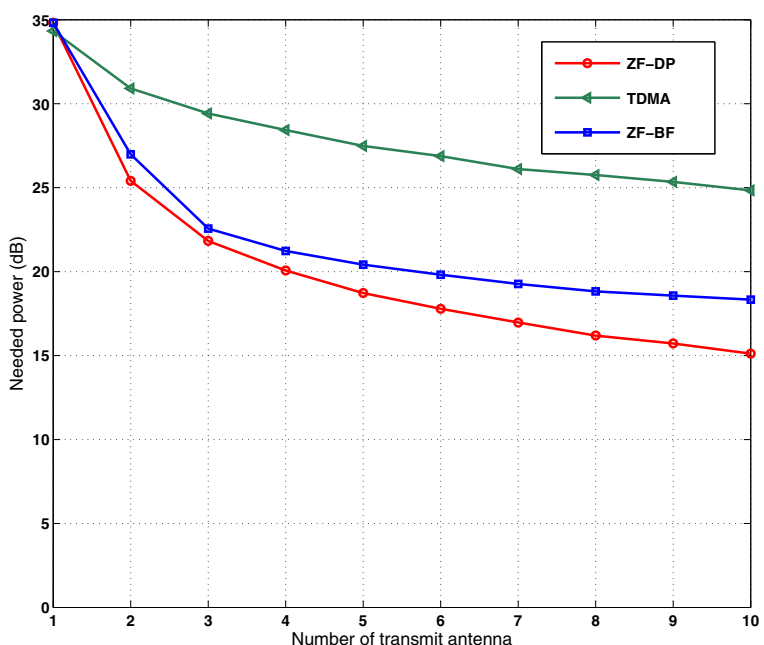

Fig. 7. The effect of the number of transmit antenna.

[3] G. Caire, S. Shamai, "On the achievable throughput of a multiantenna Gaussian broadcast channel," IEEE trans. Inf.Theory., vol. 49, no. 7, pp. 1691-1706. Jul. 2003.

[4] N. Jindal, A. Goldsmith, "Dirty-paper coding versus TDMA for MIMO broadcast channels," IEEE trans. Inf.Theory, vol. 51, no. 5, pp. 17831794. May. 2005.

[5] Q.H. Spencer, A.L. Swindlehurst and M. Haardt, "Zero-forcing methods for downlink spatial multiplexing in multiuser MIMO channels," IEEE Trans. Signal Processing, vol. 52, no.2 pp.461-471, Oct. 2004.

[6] Q.H. Spencer, C.B. Peel, A.L. Swindlehurst and M. Haardt, "An introduction to the multi-user MIMO downlink," IEEE Communications Magazine, vol. 42, no.10 pp.60-67, Oct. 2004.

[7] P. Viswanath, D.N.C. Tse, "Sum capacity of the vector Gaussian broadcast channel and uplink-downlink duality," IEEE trans. Inf.Theory., vol. 49, no. 8, pp. 1912-1921. Aug. 2003.

[8] J. Lee, N. Jindal, "Symmetric capacity of MIMO downlink channels," IEEE International Symposium on Information Theory., Seattle, WA. 2006.

[9] S. Vishwanath, N. Jindal, A. Goldsmith, "Duality, achievable rates and sum-rate capacity of MIMO broadcast channels," IEEE trans. Inf.Theory. vol. 49, no. 10, pp. 2658-2668. Oct. 2003.

[10] N. Jindal, S. Vishwanath, A. Goldsmith, "On the duality of Gaussian multiple-access and broadcast channels," IEEE trans. Inf.Theory, vol. 50, no. 5, pp. 768-783. May. 2004.

[11] H. Viswanathan, S. Venketesan, H. Huang, "Downlink capacity evaluation of cellular networks with known interference cancellation," IEEE J. Sel. Areas Commun., vol. 21, no. 5, pp. 802-811. May. 2005.

[12] H. Sato, "An outer bound on the capacity region of broadcast channel," IEEE trans. Inf.Theory, vol. 24, no. 5, pp. 374-377. May. 1978.

[13] W. Yu and J. M. Cioffi, "Sum capacity of Gaussian vector broadcast channels," IEEE trans. Inf.Theory, vol. 50, no. 9, pp. 1875-1892. Sep. 2004.

[14] P. Viswanath and D. N. C. Tse, "Sum capacity of vector broadcast channels and uplink-downlink duality," IEEE trans. Inf.Theory, vol. 49, no. 8, pp. 1912-1921. Sep. 2003.

[15] J. Oh, "Transmit power optimization for multi-user communication," $\mathrm{Ph}$.D. Dissertation, Stanford Univ., 2005.

[16] H. Weingarten, Y. Steinberg, and S. Shamai, "The capacity region of the Gaussian multiple-input multiple-output broadcast channel," IEEE trans. Inf.Theory, vol. 52, no. 9, pp. 3936-3964. Sep. 2006.

[17] G. Dimić, N. D. Sidiropoulos, "On the downlink beamforming with Greedy user selection: Performance analysis and a simple new algorithm," IEEE trans. Signal Processing, vol. 53, no. 10, pp. 3857-3868. Oct. 2005.

[18] J. Medbo and P. Schramm, "Channel models for HIPERLAN/2 in different indoors scenarios," in ETSI/BRAN 3ERI085B, Mar. 1998. 